\title{
Teor de carotenoides em nutricosméticos: análise da adequação e qualidade do produto
}

\author{
Carotenoids content in nutricosmetics products: evaluation of product \\ adequacy and quality
}

RIALA6/1572

Fernanda Marques PEIXOTO', Renata Galhardo BORGUINI², Allien Monique Rosa MACHADO², Ana Cristina Miranda Senna GÔUVEA', Sidney PACHECO², Ronoel Luiz de Oliveira GODOY²*

${ }^{*}$ Endereço para correspondência: ${ }^{2}$ Laboratório de Cromatografia Líquida, Embrapa Agroindústria de Alimentos, Empresa Brasileira de Pesquisa Agropecuária (Embrapa). Avenida das Américas 29501- Guaratiba, Rio de Janeiro, RJ, Brasil, CEP: 23020-470. Tel.: (21) 3622-9600, Fax.: (21)3622-9713. E-mail: ronoel.godoy@embrapa.br

${ }^{1}$ Programa de Pós-Graduação em Ciência e Tecnologia de Alimentos, Universidade Federal Rural do Rio de Janeiro (UFRRJ), Seropédica, RJ, Brasil

${ }^{3}$ Curso de Bacharelado em Química do Instituto Federal de Educação, Ciência e Tecnologia do Rio de Janeiro (IFRJ), Nilópolis, RJ, Brasil

Recebido: 10.05.2013 - Aceito para publicação: 14.08.2013

\section{RESUMO}

O objetivo deste trabalho foi verificar a adequação dos nutricosméticos contendo carotenoides em sua formulação em relação aos teores de $\beta$-caroteno, luteína, licopeno e zeaxantina, considerando-se as quantidades declaradas pelos fabricantes. Dezenove nutricosméticos foram adquiridos no comércio varejista do município do Rio de Janeiro, em 2012. Duas metodologias de extração foram aplicadas, variando-se de acordo com o tipo de invólucro (cápsula), veículos (excipientes) e carotenoides presentes. A quantificação e determinação do perfil de carotenoides nas amostras foram realizadas por cromatografia líquida de alta eficiência (CLAE) com método validado e acreditado. Quatro amostras das 19 analisadas estavam dentro dos limites estabelecidos pelas Boas Práticas de Fabricação (90 a 110 \%). As demais amostras revelaram conteúdo inadequado de carotenoides e, consequentemente, falhas no controle de qualidade para a produção dos nutricosméticos. Desta forma, esta pesquisa demonstra que este setor deve ser objeto de atenção especial da vigilância sanitária, com necessidade de estabelecer legislação específica para regulamentar a fabricação, a rotulagem e a propaganda destes produtos.

Palavras-chave. carotenoides, beta caroteno, CLAE.

\begin{abstract}
The present study analyzed the nutricosmetics adequacy referring to the carotenoids contents, such as $\beta$-carotene, lutein, lycopene and zeaxanthin, and these findings were compared with the contents declared by the manufacturers. Nineteen carotenoids-containing nutricosmetics were purchased at retail market in Rio de Janeiro city in 2012. Two methodologies of extraction were used according to the type of shell (capsules), carriers (excipients) and carotenoids. Quantification and determination of carotenoids profile were performed in a High Performed Liquid Chromatograph (HPLC) with validated and accredited methodology. Of 19 analyzed samples, four were within the limits established by the Good Manufacturing Practice specification (90 to $110 \%$ ), and the others were out of this range, revealing an inadequacy in the carotenoids contents and failures in the quality control of nutricosmetics production. This study demonstrated that the nutricosmetics production sector should receive a special attention from the health surveillance, and it needs a specific legislation to regulate the manufacture, labeling and advertising of these products.
\end{abstract}

Keywords. carotenoids, beta-carotene, HPLC. 


\section{INTRODUÇÃO}

Diversos estudos revelam que o ritmo de vida atual tem favorecido o aparecimento de doenças e alterações metabólicas e, como consequência, verifica-se uma forte tendência de mudança no comportamento do consumidor em relação à busca por um estilo de vida mais saudável. Esta busca por qualidade de vida, dentre outras medidas, passa pelo consumo de produtos alimentícios e não-alimentícios que promovam bem estar e saúde. Dentre os não alimentícios, estão formulações que contêm um amplo espectro de substâncias bioativas, tais como probióticos e prebióticos, vitaminas, minerais, além de metabólitos secundários de plantas, como os compostos fenólicos, carotenoides, glucosinolatos, e alcaloides, as quais são veiculadas isoladas ou/e incorporadas a extratos alimentares ${ }^{1}$. Tais produtos são comercializados sob a forma de cápsulas, soluções, géis, licores, pós e granulados, aos quais são atribuídos benefícios diretos ou indiretos à saúde. Não podem ser classificados como alimento e, portanto, são designados como um híbrido entre nutrientes e produtos farmacêuticos - os nutracêuticos ${ }^{2}$.

Segundo Zeisel ${ }^{3}$, os nutracêuticos são suplementos alimentares que proporcionam uma forma concentrada de um componente bioativo do alimento em uma matriz não alimentícia. Eles são utilizados com a finalidade de promoção da saúde em doses superiores às obtidas a partir da alimentação diária. Na Europa não existe uma regulação específica para o controle dos nutracêuticos, embora estejam sob as mesmas leis que regulam os medicamentos ${ }^{4}$. Nos EUA, a Food and Drug Administration (FDA) regula os suplementos alimentares sob diretrizes diferentes daquelas que regulamentam os alimentos e medicamentos ${ }^{2}$. No Brasil, a Agência Nacional de Vigilância Sanitária (ANVISA) os classifica como alimentos funcionais, com propriedade funcional e reivindicações de saúde. Uma vez classificados como alimentos funcionais, eles ficam sujeitos a regras específicas de rotulagem, para alegação de qualquer benefício à saúde, e inseridos na categoria de alimentos e embalagens com registro obrigatório ${ }^{5,6}$. Embora seja um termo reconhecido internacionalmente, ainda não existe um consenso sobre seu significado.

De outro modo, os alimentos funcionais são aqueles que, quando consumidos regularmente, produzem um determinado efeito benéfico à saúde, efeito este superior ao fornecido por suas propriedades nutricionais ${ }^{7}$. A fronteira entre os nutracêuticos e alimentos funcionais não é sempre clara, sendo a principal diferença a forma na qual são veiculados. Os nutracêuticos podem ser consumidos na forma de cápsulas, pastilhas, comprimidos, dentre outras, enquanto os alimentos funcionais são sempre consumidos como alimentos convencionais. Assim, quando um composto bioativo é introduzido em um determinado alimento, ele passa a ser considerado um alimento funcional. Se o mesmo composto for revestido por uma cápsula, será chamado de nutracêutico².

Recentemente, com o advento da medicina "anti-aging", que objetiva retardar o envelhecimento das células, para preservar suas funções vitais e atender aos padrões estéticos, tem surgido no mercado um novo termo; um termo mercadológico que vem sendo aplicado como sinônimo de nutracêutico. Trata-se do termo "nutricosmético" que, embora esteja associado à geração de efeitos biológicos benéficos, é direcionado aos alvos clínicos da Indústria Cosmética, tais como pele, unhas e cabelo.

Neste contexto, os nutricosméticos poderiam ser classificados como uma subcategoria dos nutracêuticos, pois suas formulações típicas também incluem um grande espectro de compostos bioativos; no entanto, sua alegação de uso vai além da saúde e envolve indicações como: reparação, prevenção, proteção solar, firmeza, pigmentação e branqueamento da pele; retenção, crescimento, restauração, nutrição e aumento de volume dos cabelos e o fortalecimento das unhas.

Alguns nutricosméticos contêm em sua formulação muitos dos carotenoides conhecidos, tais como: $\alpha$-caroteno, $\beta$-caroteno, licopeno, luteína e zeaxantina. O $\beta$-caroteno, uma provitamina $\mathrm{A}$, acumula-se na pele, proporcionando uma cor amarelo-dourada, e atua na fotoproteção contra os danos no DNA, induzidos pelos raios UV, e no combate à supressão imunitária ${ }^{8}$. A lesão cutânea é observada pelo aparecimento de manchas e foto envelhecimento, o que acelera a formação de rugas e linhas de expressão, câncer de pele e desidratação. A luteína e zeaxantina acumulamse preferencialmente na mácula lútea, protegendo a retina contra os danos foto oxidativos da luz UV.

Os nutricosméticos estão cada vez mais populares entre os consumidores, representando uma poderosa ferramenta para fins mercadológicos e, por isto, existe um grande número de produtos disponíveis no mercado ${ }^{9}$. O crescimento exponencial deste setor faz surgir a preocupação com relação a fraudes e desvios de qualidade, e com o surgimento de casos de intoxicação, bem como em evitar falsas expectativas no consumidor. Os nutricosméticos não são medicamentos, no entanto a percepção dos consumidores é dúbia. Ora, não são 
encarados como medicamentos e por isso gerariam menos efeitos colaterais, ora são levados a crer que a qualidade e a produção são compatíveis com as normas seguidas pela Indústria Farmacêutica ${ }^{10}$. Vale salientar que, embora se trate de compostos naturalmente presentes nos alimentos, a sua utilização em doses superiores às normalmente encontradas nestes podem causar efeitos adversos desconhecidos.

A composição e o conteúdo dos carotenoides nos alimentos (como em qualquer outra fonte natural) variam dependendo da estação do ano, do clima, da temperatura, da umidade do solo, e de outros fatores. Os carotenoides são altamente insaturados e propensos à isomerização e oxidação. A isomerização, destes compostos da forma trans (configuração habitual na natureza) para o isômero cis, é facilitada pelo contato com ácidos, tratamento térmico e exposição à luz ${ }^{11}$. Por isso, a coleta, identificação, manutenção da uniformidade, quantificação e padronização são fatores críticos a serem considerados ${ }^{7}$ durante as etapas de fabricação dos produtos isolados ou concentrados. Ademais, a utilização de técnicas analíticas avançadas e robustas, além de metodologia analítica validada, é outro aspecto indispensável na pesquisa e no controle de qualidade dos nutricosméticos.

Num contexto de indefinição técnica, pouca ou nenhuma normatização e fiscalização, além do mercado emergente, surgiu a presente pesquisa, cujo objetivo foi verificar a adequação dos nutricosméticos contendo carotenoides em relação ao teor de $\beta$-caroteno, luteína, licopeno e zeaxantina, considerando-se as quantidades declaradas pelo fabricante.

\section{MATERIAL E MÉTODOS}

As análises dos produtos foram realizadas no Laboratório de Cromatografia Líquida da Embrapa Agroindústria de Alimentos, que é acreditado pelo Instituto Nacional de Qualidade, Metrologia, Normatização e Industrial (INMETRO), segundo a norma ABNT NBR ISO/IEC 17025:2005, para análise de carotenoides.

Dezenove (19) produtos denominados nutricosméticos contendo carotenoides em sua formulação foram adquiridos no comércio varejista do município do Rio de Janeiro, em 2012. Cada amostra foi codificada como NC01, NC02 (...) NC19 e analisada dentro do prazo de validade. As amostras foram armazenadas em suas embalagens originais fechadas, em local fresco $\left(15\right.$ a $\left.30^{\circ} \mathrm{C}\right)$, seco e ao abrigo da luz. Os solventes utilizados foram de grau de cromatográfico e adquiridos da Tedia ${ }^{\circledR}$ Brazil, incluindo acetona, éter de petróleo, metanol $(\mathrm{MeOH})$, terc-butil metil éter (TMBE). Os reagentes para a extração de carotenoides foram adquiridos da Tedia ${ }^{\circledR}$ Brazil (Celite 545), Merck ${ }^{\circledR}$ Brasil (Hidróxido de magnésio PA), Quimex ${ }^{\circledast}$ (Sulfato de sódio anidro e cloreto de sódio) e gás nitrogênio 4,6 da White Martins ${ }^{\circledast}$. A água ultrapura $(18,2 \mathrm{M} \Omega . \mathrm{cm})$ foi produzida por sistema Milli-Q ${ }^{\circledR}$ (Millipore, Brasil). Os padrões analíticos de carotenoides foram isolados no próprio laboratório, a partir de fontes naturais, com pureza superior a $97 \%$.

\section{Extração}

Foram utilizados dois métodos de extração, variando de acordo com o tipo de invólucro (cápsula), veículos (excipientes) ecarotenoides presentes nas amostras. Utilizou-se o método descrito por Rodriguez-Amaya ${ }^{11}$ para a extração das cápsulas duras. Para as cápsulas moles, todo o conteúdo da cápsula foi dissolvido diretamente em éter etílico e/ou éter de petróleo, de acordo com os carotenoides declarados. A escolha do solvente orgânico foi feita obedecendo-se a polaridade dos compostos, se carotenos (éter de petróleo) ou xantofilas (éter etílico). Todo o procedimento de extração e análise foi realizado ao abrigo da luz e à temperatura controlada $\left(22^{\circ} \mathrm{C}\right)$, para minimizar oxidação e isomerização dos carotenoides.

\section{Análise por CLAE}

A quantificação e determinação do perfil de carotenoides nas amostras foram realizadas por Cromatografia líquida de alta eficiência (CLAE), com forno para colunas $\left(33^{\circ} \mathrm{C}\right)$, detector de rede de diodos UV-Vis (DAD 996 - Waters ${ }^{\circledR}$ ), com coluna $\mathrm{C}_{30}$ S-3 $\mathrm{YCM}^{\circledast}$ Carotenoid de 4,6 x $250 \mathrm{~mm}$. A separação foi obtida por um gradiente de eluição, com fase móvel binária de metanol/ éter metil terc-butílico, utilizando fluxo de $0,8 \mathrm{~mL} / \mathrm{min}$, volume de injeção de $15 \mu \mathrm{L}$ e tempo de corrida de 28 minutos. Os carotenoides foram identificados com base em seus tempos de retenção e de espectros de absorção UV-Vis, em comparação com os tempos de retenção dos padrões dos carotenoides. A quantificação de carotenoides totais foi realizada por espectrofotometria de UV-VIS a $450 \mathrm{~nm}$.

\section{Análise dos resultados}

Como não há legislação específica para estes produtos e visando estabelecer um critério comparativo, considerou-se satisfatória a variação dos teores entre 90 e $110 \%$ em relação ao valor declarado pelo fabricante, conforme o controle de qualidade da Indústria Farmacêutica $^{12}$. 
Peixoto FM, Borguini RG, Machado AMR, Gôuvea ACMS, Pacheco S, Godoy RLO. Teor de carotenoides em nutricosméticos: análise da adequação e qualidade do produto. Rev Inst Adolfo Lutz. São Paulo, 2013; 72(3):249-54.

Tabela 1. Descrição do produto, alegação de benefícios e recomendação de consumo, de acordo com informação de rotulagem dos nutricosméticos adquiridos no mercado varejista do Rio de Janeiro em 2012

\begin{tabular}{|c|c|c|c|}
\hline Produto & Descrição & Alegação & Recomendação de consumo \\
\hline $\mathrm{NC01}$ & $\begin{array}{c}\text { Luteína de Flor de Tagetes Erecta com vitaminas } \\
\text { e minerais }\end{array}$ & $\begin{array}{l}\text { Ação contra radicais livres e } \\
\text { antienvelhecimento }\end{array}$ & 1 cápsula ao dia \\
\hline $\mathrm{NC02}$ & $\begin{array}{c}\text { Licopeno do tomate, Lactobacillus johnsonii e } \\
\text { enriquecido com vitamina A }\end{array}$ & $\begin{array}{l}\text { Prevenção de herpes labial, queimadura solar, } \\
\text { manchas pigmentadas, xerose e intensificação } \\
\text { do bronzeado }\end{array}$ & $\begin{array}{l}1 \text { cápsula ao dia, ingerir } \\
\text { preferencialmente durante uma } \\
\text { refeição }\end{array}$ \\
\hline $\mathrm{NC03}$ & Ésteres de luteína de Flor de Tagetes Erecta & $\begin{array}{c}\text { Ação antioxidante que protegem as células } \\
\text { contra radicais livres }\end{array}$ & 1 cápsula ao dia com as refeições \\
\hline $\mathrm{NC04}$ & Luteína FloraGlo 20 \% & $\begin{array}{l}\text { Ação antioxidante que protegem as células } \\
\text { contra radicais livres }\end{array}$ & 1 cápsula ao dia \\
\hline NC05 & Licopeno do tomate & $\begin{array}{l}\text { Ação antioxidante que protegem as células } \\
\text { contra radicais livres }\end{array}$ & 1 cápsula ao dia com as refeições \\
\hline NC06 & $\begin{array}{l}\text { Licopeno do tomate enriquecido com } \\
\text { betacaroteno e vitamina } \mathrm{E}\end{array}$ & $\begin{array}{l}\text { Ação antioxidante que protegem as células } \\
\text { contra radicais livres }\end{array}$ & 1 cápsula ao dia \\
\hline NC07 & Licopeno do tomate & $\begin{array}{c}\text { Ação antioxidante e agem na formação da } \\
\text { melanina }\end{array}$ & 1 cápsula ao dia \\
\hline $\mathrm{NC08}$ & Suplemento de Vitamina A à base de beta caroteno & Ação como pró-vitamina A & 1 cápsula ao dia \\
\hline NC09 & Beta caroteno $6000 \mathrm{UI}$ & Suplementação vitamínica & $\begin{array}{l}1 \text { cápsula ao dia na principal } \\
\text { refeição }\end{array}$ \\
\hline NC10 & $\begin{array}{l}\text { Suplemento de Vitamina A à base de beta } \\
\text { caroteno }\end{array}$ & $\begin{array}{l}\text { Ação antioxidante, formação de melanina, } \\
\text { pigmentação e proteção da pele }\end{array}$ & 1 cápsula ao dia \\
\hline NC11 & Suplemento de Vitamina A e minerais & $\begin{array}{l}\text { Regeneração das células do cabelo, formação } \\
\text { de queratina e redução da queda do folículo }\end{array}$ & $\begin{array}{l}1 \text { cápsula ao dia, após uma } \\
\text { refeição ou conforme orientação } \\
\text { de nutricionista ou médico }\end{array}$ \\
\hline $\mathrm{NC} 12$ & Beta caroteno $15 \mathrm{mg}$ & Suplementação vitamínica & Não há recomendação \\
\hline NC13 & $\begin{array}{l}\text { Óleo de linhaça adicionado de licopeno de tomate, } \\
\text { luteína da flor de Tagetes Erecta, vitaminas e minerais }\end{array}$ & N.I. & 1 cápsula ao dia \\
\hline NC14 & $\begin{array}{l}\text { Licopeno com óleo de borragem enriquecido } \\
\text { com vitamina A, E e selênio }\end{array}$ & $\begin{array}{c}\text { Ação antioxidante que protegem as células } \\
\text { contra radicais livres }\end{array}$ & $\begin{array}{l}1 \text { cápsula ao dia, após uma } \\
\text { refeição ou conforme orientação } \\
\text { de nutricionista ou médico }\end{array}$ \\
\hline NC15 & $\begin{array}{c}\text { Luteína e zeaxantina da flor de Tagetes Erecta } \\
\text { com óleo de borragem enriquecido com vitami- } \\
\text { nas C e E }\end{array}$ & $\begin{array}{l}\text { Ação antioxidante que protegem as células } \\
\text { contra radicais livres }\end{array}$ & $\begin{array}{l}1 \text { cápsula ao dia, após uma } \\
\text { refeição ou conforme orientação } \\
\text { de nutricionista ou médico }\end{array}$ \\
\hline NC16 & $\begin{array}{c}\text { Licopeno, luteína e } \\
\text { zeaxantina da flor de Tagetes Erecta com ômega } \\
3 \text { de óleo de peixe enriquecidos com vitaminas } \\
\text { e minerais }\end{array}$ & $\begin{array}{c}\text { Ação antioxidante que protegem as células } \\
\text { contra radicais livres }\end{array}$ & $\begin{array}{l}1 \text { cápsula ao dia, após uma } \\
\text { refeição ou conforme orientação } \\
\text { de nutricionista ou médico }\end{array}$ \\
\hline NC17 & $\begin{array}{c}\text { Óleo de semente de groselha negra, Omega } 3 \text { de } \\
\text { óleo de peixe, licopeno de tomate enriquecido } \\
\text { com vitaminas C e E }\end{array}$ & $\begin{array}{c}\text { Atua sobre as principais causas da queda e } \\
\text { melhora a qualidade do cabelo. Fortalece as } \\
\text { unhas quebradiças }\end{array}$ & $\begin{array}{l}2 \text { cápsulas por dia durante as } \\
\text { refeições }\end{array}$ \\
\hline NC18 & $\begin{array}{l}\text { Licopeno de tomate, } \\
\text { extrato de soja enriquecido com vitamina C }\end{array}$ & $\begin{array}{l}\text { Previne e corrige a perda de firmeza da pele e } \\
\text { as rugas no rosto }\end{array}$ & $\begin{array}{l}2 \text { cápsulas por dia durante uma } \\
\text { das refeições }\end{array}$ \\
\hline NC19 & $\begin{array}{l}\text { Licopeno de tomate, zinco, vitamina E e } \\
\text { oligonutrientes essenciais }\end{array}$ & $\begin{array}{l}\text { Aumento das defesas da derme, } \\
\text { fotoproteção oral, prepara a pele para a } \\
\text { exposição solar }\end{array}$ & $\begin{array}{l}2 \text { cápsulas ao dia, } \\
\text { preferencialmente pela manhã, } \\
\text { ou conforme orientação de } \\
\text { médico e/ou nutricionista. }\end{array}$ \\
\hline
\end{tabular}

N.I. = não informado

\section{RESULTADOS E DISCUSSÃO}

As informações obtidas nas embalagens dos produtos, relativas à descrição do produto, alegação de benefícios e recomendação de consumo encontram-se na Tabela 1.
Quanto à recomendação de consumo, vale destacar que os carotenoides são compostos lipossolúveis e que a quantidade de gordura da dieta aumenta sua biodisponibilidade ${ }^{13}$, uma vez que necessitam de uma fonte lipídica para serem veiculados e, posteriormente, absorvidos pelo organismo. Desse modo, destaca-se que apenas 
Peixoto FM, Borguini RG, Machado AMR, Gôuvea ACMS, Pacheco S, Godoy RLO. Teor de carotenoides em nutricosméticos: análise da adequação e qualidade do produto. Rev Inst Adolfo Lutz. São Paulo, 2013; 72(3):249-54.

Tabela 2. Carotenoides presentes nos nutricosméticos, teor declarado pelo fabricante (em mg), teor encontrado após análise por CLAE (em $\mathrm{mg}$ ), desvio entre teor declarado e encontrado (em \%), adequação do produto em relação às BPF e preço por cápsula

\begin{tabular}{|c|c|c|c|c|c|c|}
\hline Produto & $\begin{array}{c}\text { Carotenoides no } \\
\text { produto }\end{array}$ & Teor declarado (mg) & Teor encontrado (mg) & $\operatorname{Desvio}(\%)^{* *}$ & $\begin{array}{c}\text { Adequação do } \\
\text { produto }\end{array}$ & Preço por caps (R\$) \\
\hline \multirow{2}{*}{ NC01 } & Luteína & 10 & 2,59 & $-74,1$ & \multirow{2}{*}{ Não } & \multirow{2}{*}{2,91} \\
\hline & $\beta$-caroteno* & 3,64 & 12,06 & +235 & & \\
\hline \multirow{2}{*}{$\mathrm{NCO2}$} & Licopeno & N.D. & 4,35 & N.C. & \multirow{2}{*}{ Não } & \multirow{2}{*}{3,63} \\
\hline & $\beta$-caroteno & N.D. & 0,12 & N.C. & & \\
\hline NC03 & Luteína & 20 & 12,42 & $-37,9$ & Não & 1,12 \\
\hline NC04 & Luteína & 10 & 9,1 & -9 & Sim & 1,83 \\
\hline NC05 & Licopeno & 10 & 12,42 & $+24,2$ & Não & 1,22 \\
\hline \multirow{2}{*}{ NC06 } & Licopeno & 5,1 & 1,57 & $-69,22$ & \multirow{2}{*}{ Não } & \multirow{2}{*}{1,43} \\
\hline & $\beta$-caroteno & 3,6 & 3,83 & $+6,39$ & & \\
\hline NC07 & Licopeno & 5 & 4,82 & $-3,6$ & Sim & 1,36 \\
\hline NC08 & $\beta$-caroteno & 4 & 2,68 & -33 & Não & 0,85 \\
\hline NC09 & $\beta$-caroteno & 3,64 & 3,17 & $-12,91$ & Sim & 0,54 \\
\hline $\mathrm{NC} 10$ & $\beta$-caroteno & 3,64 & 3,45 & $-5,22$ & Sim & 1,61 \\
\hline NC11 & $\beta$-caroteno & 3,64 & 4,76 & $+30,77$ & Não & 3,81 \\
\hline NC12 & $\beta$-caroteno & 15 & 15,97 & $+6,47$ & Sim & 0,47 \\
\hline \multirow{3}{*}{ NC13 } & Licopeno & 5,1 & 1,25 & $-75,49$ & \multirow{3}{*}{ Não } & \multirow{3}{*}{3,00} \\
\hline & Luteína & 4 & 2,8 & -30 & & \\
\hline & $\beta$-caroteno* & 3,64 & 5,01 & $+39,17$ & & \\
\hline \multirow{2}{*}{ NC14 } & Licopeno & 6 & 6,22 & $+3,67$ & \multirow{2}{*}{ Não } & \multirow{2}{*}{3,55} \\
\hline & $\beta$-caroteno ${ }^{*}$ & 3,64 & 4,92 & $+36,67$ & & \\
\hline \multirow{2}{*}{ NC15 } & Luteína; & 10 & 18,22 & $+82,2$ & \multirow{2}{*}{ Não } & \multirow{2}{*}{3,81} \\
\hline & Zeaxantina & 0,5 & 1,34 & +168 & & \\
\hline \multirow{3}{*}{ NC16 } & Licopeno & 6 & 2,92 & $-51,33$ & \multirow{3}{*}{ Não } & \multirow{3}{*}{3,81} \\
\hline & Luteína & 10 & 13,77 & $+37,7$ & & \\
\hline & Zeaxantina & 0,5 & 1,08 & +116 & & \\
\hline NC17 & Licopeno & N.D. & 1,52 & N.C. & Não & 2,00 \\
\hline NC18 & Licopeno & N.D. & 2,1 & N.C. & Não & 2,07 \\
\hline NC19 & Licopeno & 10 & 0,016 & $-99,84$ & Não & 1,65 \\
\hline
\end{tabular}

N.D.: não declarado

N.C.: não calculado

*Os valores declarados como $600 \mu \mathrm{g}$ de vitamina A (equivalente a 100 \% da Ingestão Diária Recomendada) foram convertidos para $3,64 \mathrm{mg}$ de $\beta$-caroteno

${ }_{* \star}$ Percentual de carotenoides encontrado em relação ao conteúdo declarado pelo fabricante

$58 \%$ dos fabricantes orientam corretamente o consumidor quanto ao consumo do produto durante as refeições (Tabela 1) para viabilizar a absorção dos carotenoides.

Os dados relativos aos tipos de carotenoides presentes nos nutricosméticos, ao teor declarado pelo fabricante, ao teor encontrado após análise por CLAE, ao desvio entre teor declarado e encontrado e à avaliação da adequação em relação às BPF foram apresentados na Tabela 2. Além disso, apresenta-se o preço por cápsula de cada produto, variando de $\mathrm{R} \$ 0,47$ a 3,81, que foi calculado a partir do atual valor de mercado e da quantidade de cápsulas presentes em cada embalagem.

Ao se comparar os resultados obtidos nas análises com os valores declarados (quando informado), observou- se que quatro das dezenove amostras se encontravam dentro do limite estabelecido como adequado (90 a $110 \%$ ). Vale salientar que três amostras (NC02, NC17 e NC18) não apresentavam declaração de concentração de carotenoides no rótulo, embora seja uma exigência da ANVISA, e que, portanto, a análise comparativa não foi possível, embora os carotenoides tenham sido quantificados.

Em duas amostras (NC06 e NC14) pelo menos um dos carotenoides declarados apresentava-se dentro dos limites estabelecidos, enquanto que os demais estavam fora deste limite. No entanto, como resultado de um dos carotenoides estava fora, desqualificou-se a amostra de uma forma geral. Vale destacar também dois resultados bastante extremos, onde as amostras apresentaram teores 
superiores a 168 e $235 \%$ do conteúdo declarado (NC01 e $\mathrm{NC15}$, respectivamente). Aman et $\mathrm{al}^{14}$ verificaram que das onze amostras testadas de suplementos contendo carotenoides, todas apresentaram dosagens até $45 \%$ superiores às declaradas. Isto sugere que alguns fabricantes, por conhecerem a baixa estabilidade destes compostos, praticam a superdosagem a fim de compensar possíveis perdas durante $\mathrm{o}$ armazenamento. Além disso, uma amostra avaliada apresentou resultado 99,8 \% inferior ao declarado, demonstrando pouco ou nenhum controle do processo de produção. Vale salientar que certos produtos, que declaram apresentar carotenoides, tais como a luteína e a zeaxantina, não foram capazes de demonstrar compatibilidade com sua rotulagem, por razões já conhecidas entre os pesquisadores da área ${ }^{15}$, tal como a dificuldade da seleção de boas fontes naturais (alimentos e/ou plantas) para o isolamento destes compostos. Assim, para exemplificar a dificuldade mencionada, um dos fabricantes declarou que o produto ( $\mathrm{NC01}$ ) continha altos teores de luteína e menores de $\beta$-caroteno, no entanto, ao se analisar o produto, constatou-se a ocorrência da inversão nas concentrações, já que foram encontrados alto valor do $\beta$-caroteno (235\% acimado do declarado) e baixo valor da luteína (74\% inferior ao declarado). Este fato pode ser explicado pela maior disponibilidade de fontes naturais para a obtenção de $\beta$-caroteno, em detrimento da luteína. A maior abundância deste composto também pode ser confirmada pela presença do mesmo em amostras em que o $\beta$-caroteno não havia sido declarado, tal como a amostra NC16.

Ademais, não foi observada relação entre os preços praticados pelos fabricantes e a qualidade dos produtos. O produto $\mathrm{NC12}$, embora apresentasse maior nível de equivalência de teor, tinha o menor custo por cápsula. Enquanto o NC19, que obteve o pior resultado comparativo, apresentou um elevado custo por cápsula, demonstrando não haver relação direta entre o preço e a qualidade dos produtos.

Os nutracêuticos têm sido considerados como um fenômeno de mercado e suas vendas crescem expressivamente. Os resultados encontrados indicaram que o número de produtos inadequados, que exibiram alto desvio em relação ao teor de carotenoides declarado no rótulo, é relevante (aproximadamente $80 \%$ ) e que o setor deve ser objeto de atenção especial da vigilância sanitária, havendo a necessidade de legislação específica para regulamentação da fabricação, da rotulagem e da propaganda desses produtos. Além disso, os profissionais da área de saúde, que fazem a prescrição destes produtos, devem ser alertados quanto aos riscos da falta de controle pelos fabricantes que, além de declararem o seu conteúdo erroneamente, demonstraram não conformidade quanto às Boas Práticas de Fabricação (BPF). Cabe concluir que a falta de controle de qualidade na fabricação destes produtos, que são consumidos na expectativa de apresentarem efeitos benéficos à saúde e à estética, impacta diretamente em sua eficácia e segurança, podendo representar um potencial risco ao consumidor.

\section{REFERÊNCIAS}

1. Lockwood GB. The quality of commercially available nutraceutical supplements and food sources. J Pharm Pharmacol. 2011;63(1):3-10.

2. Espín JC, García-Conesa MT, Tomás-Barberán FA. Nutraceuticals: facts and fiction. Phytochem. 2007; 68(22-24): 2986-3008.

3. Zeisel SH. Regulation of "Nutraceuticals". Science.1999; 285(5435):1853-5.

4. Coppens P, Silva MF, Silva PS. European regulations on nutraceuticals, dietary supplements and functional foods: A framework based on safety. Toxicol. 2006; 221(1):59-74.

5. Brasil. Ministério da Saúde. Resolução RES $n^{\circ} 18$, de 30 de abril de 1999. Aprova o regulamento técnico que estabelece as diretrizes básicas para análise e comprovação de propriedades funcionais e ou de saúde alegadas em rotulagem de alimentos. Diário Oficial [da] República Federativa do Brasil. Brasília, DF, 03 mai. 1999. Seção 1, n³98. p.11.

6. Brasil. Ministério da Saúde. Resolução RES no 19, de 30 de abril de 1999. Aprova o regulamento técnico de procedimentos para registro de alimento com alegação de propriedades funcionais e ou de saúde em sua rotulagem. Diário Oficial [da] República Federativa do Brasil. Brasília, DF, 03 mai. 1999. Seção 1, n³99. p.12.

7. Bernal J, Mendiola JA, Ibañez E, Cifuentes, A. Advanced analysis of nutraceuticals. J Pharm Biomed Anal. 2011;55(4): 758-74.

8. Anunciato TP, Filho PAR. Carotenoids and polyphenols in nutricosmetics, nutraceuticals, and cosmeceuticals. J Cosmet Dermatol. 2010;11(1):51-4.

9. Bagchi D. Nutraceutical and funcional food regulations in the United States around the world. Toxicol. 2006;221(1): 1-3.

10. Rossi D, Guerrini A, Bruni R, Brognara E, Borgatti M, Gambari $\mathrm{R}$, et al. Trans-Resveratrol in nutraceuticals: Issues in retail quality and effectiveness. Molecules. 2012;17(10):12393-405.

11. Rodriguez-Amaya DB. A guide to carotenoids analysis in foods. Washington (DC): International Life Sciences Institute (ILSI) Press; 1999.

12. Farmacopeia Brasileira. $4^{\mathrm{a}}$ ed. São Paulo: Atheneu; 1996.

13. Yonekura L, Nagao A. Intestinal absorption of dietary carotenoids. Mol Nutr Food Res. 2007;51(1):107-15.

14. Aman R, Bayha S, Carle R, Schieber A. Determination of carotenoid stereoisomers in comercial dietary supplements by High performance Liquid Chromatography. J Agric Food Chem. 2004;52(20):6086-90.

15. Kimura M, Rodrigueza-Amaya DB. A scheme for obtaining standards and HPLC quantification of leafy vegetable carotenoids. Food Chem.2002;78(3): 389-98. 